\title{
Manejo práctico de una eosinofilia
}

\author{
J. L. PÉREZ-ARELLANO ${ }^{1,2}$, J. PARDO ${ }^{3}$, M. HERNÁNDEZ-CABRERA ${ }^{1}$, \\ C. CARRANZA ${ }^{2,3}$, A. ÁNGEL-MORENO ${ }^{1,2}$, A. MURO ${ }^{3}$ \\ ${ }^{1}$ Unidad de Enfermedades Infecciosas y Medicina Tropical. Servicio de Medicina Interna. \\ Hospital Universitario Insular de Las Palmas. ${ }^{2}$ Departamento de Ciencias Médicas y \\ Quirúrgicas. ${ }^{3}$ Laboratorio de Parasitología. Facultad de Farmacia. Universidad de \\ Salamanca
}

\author{
EOSINOPHILIA: A PRACTICAL APPROACH
}

\begin{abstract}
RESUMEN
En esta revision se define inicialmente el concepto y grado de eosinofilia. En un segundo apartado se estudian las causas principales de esta alteración hematológica considerando tres tipos de pacientes: a) autóctonos no infectado por VIH; b) infectados por VIH; y c) procedentes de regiones tropicales (eosinofilia importada). En tercer lugar, se desarrolla un protocolo diagnóstico secuencial, indicando las pruebas mas útiles para llegar al diagnóstico en los grupos mencionados previamente. Específicamente, se incluyen las pruebas complementarias útiles en los pacientes con eosinofilia importada, tanto en presencia como en ausencia de datos de focalidad. Tras una breve referencia a las consecuencias patológicas de la eosinofilia, se contemplan las mediadas terapéuticas útiles en estos pacientes. En este ultimo apartado se hace un especial énfasis en el tratamiento antihelmíntico y en el manejo del síndrome hipereosinofílico.
\end{abstract}

PALABRAS CLAVE: Eosinofilia. Enfermedades importadas. Helmintos. Diagnóstico. Tratamiento.

\begin{abstract}
In this first part of this paper we review the definition of eosinophilia and their classification according to the degree of elevation of eosinophils/ $\mu$ L. Aetiological factors related with eosinophilia were described in three groups of patients: a) autochthonous non-infected by HIV; b) HIVinfected; and c) arrived from tropical countries (imported eosinophilia). We included an algorithmic approach to the diagnosis, including the diagnostic studies that should be performed in patients with or without organ involvement. Pathological consequences of eosinophilia are indicated in the next part of the paper. Finally, therapeutical options used in patients with eosinophilia are reviewed, with an special emphasis on antihelminthic therapies and the management of the hypereosinophilic syndromes.
\end{abstract}

KEY WORDS: Eosinophilia. Imported diseases. Helminths. Diagnosis. Treatment.

Pérez-Arellano JL, Pardo J, Hernández-Cabrera M, Carranza C, Angel-Moreno A, Muro A. Manejo práctico de una eosinofilia. An Med Interna (Madrid) 2004; 21: 244-252.

\section{INTRODUCCIÓN}

La eosinofilia es un dato que aparece de forma relativamente frecuente en la práctica clínica. Su importancia radica en dos aspectos principales: diagnósticos y patogénicos. Así, desde un punto de vista diagnóstico, la detección de eosinofilia sugiere la presencia de diversas entidades. Por otro lado, en algunas circunstancias, la liberación de productos de estos leucocitos se asocia a lesiones tisulares.

Aunque los mecanismos fisiopatológicos por los que aparece eosinofilia son de gran interés (1), en esta revisión no serán contemplados de forma detallada debido a razones de espacio. Inicialmente se revisará el concepto de eosinofilia, indicando sus limitaciones y nomenclatura. En un segundo apartado se consideran, de forma detallada, las principales causas de eosinofilia agrupadas desde un punto de vista práctico. El siguiente epígrafe revisa las consecuencias patológicas de la eosinofilia tanto como dato "de alarma" como debidas directamente a la liberación de sustancias. Finalmente se consideran los principales aspectos terapéuticos, haciendo especial énfasis en el manejo de los antihelmínticos y en el tratamiento de la eosinofilia idiopática.

\footnotetext{
Trabajo aceptado: 8 de enero de 2004
} 


\section{CONCEPTO Y LÍMITES}

Se considera que existe eosinofilia cuando el número total de eosinófilos circulantes en sangre periférica es significativamente superior al presente en la población normal. Los valores descritos por diferentes autores son muy variables oscilando entre 350 y $700 / \mu \mathrm{L}$. Así, Rothemberg considera como límite para definir esta situación el de 350 eosinófilos/ $\mu \mathrm{L}$ (1), mientras que Bridgen considera que existe eosinofilia a partir de $700 / \mu \mathrm{L}$ (2). De cualquier forma, la mayor parte de autores consideran que existe eosinofilia cuando el número de eosinófilos es igual o mayor a $450 / \mu \mathrm{L}(3,4)$.

Sin embargo, este valor numérico debe ser matizado en relación con los datos clínicos, ya que algunos fármacos o diferentes situaciones (tanto fisiológicas como patológicas) pueden modificar esta magnitud. Entre los factores fisiológicos que influyen en el número de eosinófilos circulantes se encuentran los siguientes: la hora del día en la que se realice la extracción de la muestra, la edad, el sexo, y el embarazo. A modo de ejemplo, la variación diurna del número de eosinófilos puede ser de hasta un $40 \%$, mayor por la noche que por la mañana, en relación con el ritmo circadiano de corticoides. También influye en esta magnitud el empleo de fármacos (p. ej. adrenalina, $\beta$-bloqueantes o corticoides) que puede modificar de forma notable el número de eosinófilos (hasta en un $30 \%$ ). Finalmente, la presencia de infecciones agudas (principalmente víricas, bacterianas o protozoarias, especialmente la malaria) puede disminuir transitoriamente el número de eosinófilos circulantes, reapareciendo los valores basales una vez resuelto el problema infeccioso que ocasionó este descenso. Es importante insistir en que una infección no helmíntica puede llevar, de forma transitoria, a "normalizar" valores de eosinófilos muy elevados. Esta circunstancia puede observarse, en la práctica, en situaciones de patología importada (específicamente en el paciente con malaria) en las que, una vez resuelta la infección protozoaria, se detecta eosinofilia.

Aunque no ha sido estudiado adecuadamente, el aumento del tanto por ciento de eosinófilos sin aumento del número total, no debe ser considerado de forma práctica una eosinofilia.

\section{GRADOS DE EOSINOFILIA}

En la práctica clínica, se acepta que el número total de eosinófilos tiene valor en la orientación diagnóstica, de tal forma que a medida que el número de eosinófilos es mayor, disminuyen las posibilidades diagnósticas. Sin embargo, no existen trabajos rigurosos en los que se relacione el grado de eosinofilia con los determinados agentes etiológicos.

De cualquier forma, la mayor parte de los autores emplea como puntos de corte los valores de 1000 y $3000 / \mu \mathrm{L}$ para definir 3 grados de eosinofilia. Así, se consideraría eosinofilia leve entre 450 y 999 eosinófilos/ $\mu \mathrm{L}$; eosinofilia moderada entre 1000 y 2999 eosinófilos/ $\mu \mathrm{L}$ y eosinofilia intensa cuando las cifras de eosinófilos superen los 3000/ $\mu \mathrm{L}$ (4).

\section{SIGNIFICADO CLÍNICO DE UNA EOSINOFILIA}

Una regla práctica indica que la detección de eosinofilia en una persona que proceda o haya viajado a una región tropi- cal debe hacer pensar en primer lugar en causas infecciosas, mientras que en una persona que no haya presentado este dato epidemiológico deben descartarse otro tipo de causas (inmunológicas, farmacológicas, neoplásicas o endocrinas). Una situación especial aparece en los pacientes infectados por el VIH, tanto debido a la propia naturaleza de la infección como a los fármacos utilizados en su control o a presencia de enfermedades asociadas. Por ello, de forma operativa podemos distinguir tres situaciones diferentes en un paciente con eosinofilia.

\section{EOSINOFILIA EN EL PACIENTE AUTÓCTONO NO INFECTADO POR} $\mathrm{VIH}$

En nuestro país, las principales causas pueden incluirse en siete grupos: (i) farmacológicas (ii) alérgicas, principalmente reacciones de hipersensibilidad de tipo I (iii) inmunológicas no alérgicas (iv) neoplasias (v) alteraciones endocrinas y metabólicas (vi) parasitarias (vii) trastornos idiopáticos.

Las causas farmacológicas de eosinofilia son múltiples y presumiblemente se incrementarán a medida que se incorporen nuevos principios activos al arsenal terapéutico. Por ello, en cualquier paciente con eosinofilia, es esencial conocer de forma precisa la medicación que recibe, ya que algunos fármacos (incluso aquellos de empleo muy común) pueden ser los responsables de esta alteración analítica. En la tabla I se recogen los fármacos más importantes, aunque somos conscientes de que el listado nunca puede ser exhaustivo (2,5-10). Tiene interés señalar que la eosinofilia relacionada con fármacos, en ocasiones no sólo es un dato analítico sino que puede formar parte de un cuadro clínico más grave en el que también aparecen manifestaciones sistémicas (p. ej. fiebre, síndrome constitucional) o locales (p. ej. exantema, infiltrados pulmonares, nefropatía, hepatopatía, etc.). Por otro lado, aunque la eosinofilia farmacológica no suele asociarse a atopia o a historia familiar, tanto en los pacientes con hepatopatía o nefropatía previa, como en los acetiladores lentos, es más frecuente que la población general (2).

Las reacciones de hipersensibilidad no farmacológicas mediadas por IgE son una causa muy frecuente de eosinofilia. Las más frecuentes son las rinitis (de las que existen diferentes variedades) y algunas formas de asma. También se incluirían en este grupo algunas respuestas inmunológicas a microorganismos, principalmente la aspergilosis bronco-pulmonar alérgica (11). En estas situaciones, los datos clínicos y los exámenes complementarios suelen permitir realizar un diagnóstico simple. En casos dudosos, la realización de estudios específicos (p. ej. la demostración de eosinófilos en la mucosa nasal, las pruebas de provocación bronquial o la medida de anticuerpos específicos) permiten el diagnóstico final.

También existen otras enfermedades inmunológicas no alérgicas se asocian a eosinofilia. Desde un punto de vista patogénico estas entidades son de tres tipos: enfermedades autoinmunes órgano-inespecíficas, autoinmunopatías órganoespecíficas e inmunodeficiencias. Dentro de las enfermedades autoinmunes sistémicas, las que con mayor frecuencia presentan eosinofilia son la enfermedad de Churg-Strauss $(12,13)$ y la fasciitis eosinofílica (síndrome de Shulman) (14), una enfermedad similar a la esclerodermia (pero en la que la epidermis y dermis están respetadas), caracterizada por un inicio agudo, muy frecuentemente tras un traumatismo, con eritema 
TABLA I

FÁRMACOS RELACIONADOS CON EOSINOFILIA

\begin{tabular}{l} 
Fármacos empleados en infecciones \\
\hline Penicilinas \\
Cefalosporinas \\
Glucopéptidos \\
Sulfamidas (Cotrimoxazol) \\
Tetraciclinas (sobre todo Minociclina) \\
Quinolonas (descrita en Ciprofloxacino y Norfloxacino) \\
Antituberculosos (sobre todo Rifampicina y Etambutol) \\
Nitrofurantoína \\
Antimaláricos (sobre todo Fansidar ${ }^{\circledR}$, excepcionalmente cloroquina) \\
Fármacos empleados en enfermedades cardiovasculares
\end{tabular}

Inhibidores de la ECA (efecto de clase)

Espironolactona

Diltiacem

Quinidina

Alfa metil dopa

Fármacos empleados en enfermedades neurológicas

Antipsicóticos (cloropromazina, olanzapina)

Antidepresivos (imipramina, desimipramina trazodona, triptofano[síndrome eosinofilia/mialgia])

Anticonvulsivantes (difenilhidantoína, carbamacepina, fenobarbital, valproato)

Fármacos antiinflamatorios y antireumáticos

Antiinflamatorios no esteroideos no esteroideos (efecto de clase)

Sales de oro

Fármacos empleados en enfermedades digestivas

Antagonistas $\mathrm{H} 2$ (ranitidina)

Inhibidores de la bomba de protones (omeprazol, lansoprazol)

Aminosalicilatos (sulfasalacina, mesalacina)

Fármacos empleados en enfermedades neoplásicas

Múltiples (bleomicina, metotrexato, procarbacina, fludarabina)

Otros

Hipoglucemiantes orales "clásicos" (Clorpropamida, Tolbutamida)

Anticoagulantes (Heparina sódica, Enoxaparina)

Hipolipemiantes (Colestiramona)

Hipouricemiantes (Alopurinol)

Anestésicos (Halotano)

Miorrelajantes (Dantroleno)

y edema asociado posteriormente a induración en la piel de las extremidades. Con mucho menor frecuencia, aparece eosinofilia en otras enfermedades sistémicas como las formas graves de artritis reumatoide, o la granulomatosis de Wegener. Las formas localizadas de enfermedad autoinmune pueden afectar a diversos órganos o sistemas (Tabla II). Así la afectación pulmonar de origen inmune es característica de la neumonía eosinófila (tanto crónica como aguda) $(15,16)$. La afectación cutá-
TABLA II

ENFERMEDADES INMUNOLÓGICAS CON EOSINOFILIA Y DATOS FOCALES

Pulmonares

Neumonía eosinofílica crónica

Neumonía eosinófila aguda

Cutáneas

Eccema

Dermatitis herpetiforme

Pénfigo

Penfigoide bulloso

Enfermedad de Kimura

Hiperplasia angiolinfoide con eosinofilia

Síndrome de Wells

Síndrome de Gleich

Síndrome NERDS

Hematológicas

Anemia perniciosa

Digestivas

Enteritis eosinofilica

Enfermedad inflamatoria crónica intestinal

Neurológicas

Meningitis eosinofílica idiopática

Urológicas

Cistitis eosinofílica

Inicio de hemodiálisis

Inicio de diálisis peritoneal

nea de origen inmunológico asociada a eosinofilia aparece en varias situaciones como: algunas formas de eczema, la dermatitis herpetiforme, el pénfigo, el penfigoide bulloso, la enfermedad de Kimura (grandes masas subcutáneas en cuello o cabeza en pacientes procedentes del sudeste asiático) (17), la hiperplasia angiolinfoide con eosinofilia (lesiones superficiales más pequeñas que las de la enfermedad de Kimura) (18), el síndrome de Wells (celulitis eosinofílica, con imágenes características en la histología en "forma de llama") (19), el síndrome de Gleich (episodios recurrentes de angioedema, urticaria, prurito, fiebre, aumento de peso, aumento de IgM y eosinofilia) (20) y el síndrome NERDS (nodules, eosinophilia, rheumatism, dermatitis, swelling; es decir nódulos paraarticulares prominentes, urticaria recurrente con con angioedema y eosinofilia tisular) (21). La principal enfermedad del sistema hematopoyético no maligna que se asocia a eosinofilia es la anemia perniciosa (22). Otras enfermedades localizadas con eosinofilia afectan al tubo digestivo (p. ej. enfermedad inflamatoria crónica intestinal y/o enteritis eosinofílica) $(23,24)$, al sistema nervioso (meningitis eosinofílica) (25) o genitourinario (cistitis eosinofilica (26), hemodiálisis (27) o diálisis peritoneal (28).

Las inmunodeficiencias que con mayor frecuencia se asocian a eosinofilia son el síndrome de Job (29) y la enfermedad 
de Omenn (30). Más rara vez aparece en el síndrome de Wiskott-Aldrich y en la deficiencia selectiva del IgA (en este caso asociada a fasciitis eosinofilica).

En diversas neoplasias puede aparecer eosinofilia, principalmente en la enfermedad de Hodgkin (hasta en un $15 \%$ de casos) y en otras neoplasias hematológicas, en concreto leucemias (sobre todo con fenotipo M4) y linfomas (sobre todo de estirpe B (31). En lo que respecta a los tumores sólidos, es más frecuente en las neoplasias de células grandes no queratinizadas de cuello, carcinomas indiferenciados de células grandes de pulmon, carcinomas epidermoides de pene, vagina, piel y nasofaringe, adenocarcinomas de estómago, colon y endometrio así como en el carcinoma transicional de vejiga.

Las dos principales enfermedades endocrino-metabólicas en las que puede aparecer esta alteración hematológica son la insuficiencia suprarrenal (crónica y aguda) (32) y el embolismo por cristales de colesterol (33).

Aunque las helmintosis son mucho más frecuentes en el paciente procedente de regiones tropicales o que ha viajado a las mismas, no debe olvidarse que existen algunas parasitosis autóctonas capaces de producir eosinofilia $(34,35)$. Los helmintos autóctonos que con mayor frecuencia son capaces de ocasionar eosinofilia se indican en la tabla III.

\section{TABLA III}

PARASITOSIS AUTÓCTONAS CAUSANTES DE EOSINOFILIA

Anisakis sp.

Echinococcus granulosus

Fasciola hepática

Strongyloides stercoralis

Taenia sp.

Toxocara canis

Trichinella sp.

Trichostrongylus sp.

Finalmente existen algunos procesos de causa desconocida en los que la eosinofilia es un dato importante. Las principales situaciones son el síndrome hipereosinofílico idiopático (36) la eosinofilia hereditaria (37) y la eosinofilia idiopática adquirida. El síndrome hipereosinofílico es un conjunto heterogéneo de trastornos que se definen en la práctica por la asociación de cuatro datos: a) eosinofilia superior a 1500 eosinófilos; b) persistencia durante más de 6 meses; c) evidencia de afectación de órganos y d) exclusión de otras causas capaces de ocasionar eosinofilia. Los dos primeros criterios requieren una eosinofilia importante (moderada o intensa) y la persistencia de la misma, descartando de esta forma eosinofilias transitorias. El tercer criterio implica la afectación de algún órgano o sistema. Los principales órganos lesionados en el síndrome hipereosinofílico son (i) corazón, apareciendo afectación endomiocárdica que por formación de trombos y cicatrización puede llevar a la insuficiencia cardiaca (ii) sistema nervioso central. lo que puede manifestarse por fenómenos isquémicos, encefalopatía difusa y/o neuropatía periférica (iii) pulmón, cuya manifestación característica es la tos asociada o no a infiltrados pulmonares, pero sin asma (iv) piel, tanto en forma de urticaria /angioedema como por pápulas pruriginosas y (v) bazo, cuya manifestación característica es la esplenomegalia. Finalmente, deben excluirse otras entidades capaces de desencadenar una eosinofilia prolongada. Como en todos los síndromes idiopáticos, este último criterio es complejo de aplicar ya que las pruebas precisas para excluir "todos" los agentes conocidos no ha sido adecuadamente definida. Por ello, en esta entidad se incluyen al menos tres tipos de procesos: eosinofilias reactivas a procesos no identificados, trastornos clonales linfoides que ocasionan eosinofilia reactiva a través de citocinas y trastornos clonales de la serie mieloide $(38,39)$. Evidentemente, en los dos últimos casos no deben cumplirse los criterios diagnósticos de neoplasias linfoides o mieloides que constituirían causas secundarias. La eosinofilia familiar es una situación excepcional, caracterizada por una herencia autosómica dominante y en la que existen datos de afectación cardiaca o neurológica (37). Finalmente, existen casos de eosinofilia prolongada, sin causa explicable en la que no existe evidencia de afectación de órganos, por lo que conceptualmente no pueden ser incluidos dentro del síndrome hipereosinofílico y que pueden ser definidos como eosinofilia idiopática adquirida.

\section{EOSINOFILIA EN EL PACIENTE AUTÓCTONO INFECTADO POR VIH}

La alteración inmunológica característica de la infección por VIH es la disminución de los linfocitos CD4. Las modificaciones del fenotipo linfocitario se han asociado a un incremento en el número de eosinófilos a medida que avanza la inmunodepresión (40), apareciendo, en algunas series hasta en un $12,6 \%$ de los pacientes (41). En nuestro país no existen estudios detallados acerca de este aspecto, aunque la impresión generalizada es que la presencia de eosinofilia en los individuos infectados por el VIH es menor que la descrita en las publicaciones citadas. De hecho, algunos autores sugieren que los estudios dirigidos a aclarar el mecanismo de la eosinofilia no aporta resultados en los pacientes con infección VIH (42). Sin embargo, existen algunas causas específicas de eosinofilia que deben ser consideradas de forma particular en este grupo. En concreto, debieran valorarse las siguientes posibilidades: (i) toxicidad medicamentosa (particularmente nevirapina, efavirenz y cotrimoxazol) (43-45) (ii) empleo de fármacos estimulantes de la hemopoyesis (GM-CSF) (iii) insuficiencia suprarrenal por citomegalovirus (46) (iv) parasitosis (Isospora belli, Strongyloides stercoralis) y (v) foliculitis eosinofílica (47).

\section{EOSINOFILIA IMPORTADA}

Como se ha señalado previamente, la presencia de eosinofilia en este contexto es casi exclusiva de las infecciones por helmintos. De forma excepcional, puede aparecer esta alteración analítica en algunas infecciones bacterianas (p. ej. resolución de una escarlatina o en formas crónicas de tuberculosis o lepra), enfermedades víricas (resolución de una infección vírica o infección VIH), micosis (especialmente en la coccidioidomicosis) (48) o algunas protozoosis concretas (Isospora belli, Dientamoeba fragilis, Sarcocystis sp y Blastocystis hominis) (49-52).

Planteado de esta forma, el diagnóstico de eosinofilia aparentemente resulta sencillo, aunque en la práctica debemos realizar algunas matizaciones $(2,53-58)$ : 
-No todos los helmintos inducen eosinofilia del mismo grado. Así, se distinguen varios patrones de afectación como (a) la ausencia de eosinofilia (p. ej. hidatidosis no complicada); (b) formas fluctuantes (asociadas a los movimientos del parásito en los tejidos: Loa loa, Dracunculus medinensis, Gnathostoma spinigerum); (c) elevada durante toda la infección (p. ej. Toxocara canis, Trichinella spiralis); (d) limitada a un estadio parasitario (p. ej. fase larvaria de Ascaris lumbricoides); (e) de intensidad variable atendiendo a las diferentes fases de la parasitosis (p. ej esquistosomosis, estrongiloidosis o uncinariosis) o (f) presente tras un proceso intercurrente o durante el tratamiento (rotura de un quiste hidatídico, tratamiento de una filariosis).

- El estudio coproparasitario, prueba básica en el diagnóstico de las parasitosis intestinales, posee una baja sensibilidad en el diagnóstico de eosinofilia. Así, por ejemplo, en el diagnóstico de estrongiloidosis, incluso empleando técnicas coprológicas dirigidas, la sensibilidad es menor del $50 \%$.

- Un mismo paciente pueden presentar simultáneamente varias parasitosis, siendo su participación en la aparición de eosinofilia diferente. Así, por ejemplo, la detección en heces de Trichuris trichura (que por sí mismo ocasiona exclusivamente una eosinofilia leve) asociada a valores elevados de eosinófilos obliga a la búsqueda de otros agentes causales.

-Es muy importante considerar los aspectos geográficos, ya que determinadas parasitosis poseen una distribución localizada. Así, por ejemplo, no debe considerarse la posibilidad de una infección por Loa loa en países diferentes de los del centro de Africa o una infección por Clonorchis sinensis u Opistorchis viverrini en personas que nunca han estado en el Sudeste asiático.

\section{PROTOCOLO DIAGNÓSTICO ANTE UN PACIENTE CON EOSINOFILIA}

El estudio de un paciente con eosinofilia debe realizarse de forma ordenada, empleando pruebas sencillas en una primera fase, que permitirán un diagnóstico directo en muchos casos y, en el resto permitirán clasificar al paciente en los tres grupos indicados previamente.

La fase inicial incluirá una anamnesis y exploración física completa, un hemograma (que identificará y permitirá conocer el grado de eosinofilia, además de evaluar las otras series), un estudio bioquímico (que incluya evaluación hepática, renal y muscular), una radiografía de tórax, un sistemático y sedimento de orina y un estudio coproparasitario (tres muestras). En presencia de factores de riesgo o datos clínicos sugerentes, deberá solicitarse una serología frente a VIH, con la autorización del paciente (Fig. 1).

Tras la aplicación de estas pruebas es posible realizar un diagnóstico etiológico o al menos sugerir las pruebas necesarias para realizarlo. Así, por ejemplo, ante la sospecha de una eosinofilia farmacológica deberá suspenderse el o los fármacos potencialmente implicados, añadiendo eventualmente corticoides si el cuadro clínico es grave. Por otro lado, la presencia de datos localizadores (pulmonares, cutáneos, osteoarticulares, etc.) llevará a los estudios complementarios pertinentes (estudios de imagen, endoscopia, biopsia tisular). En otros casos, tras la aplicación de este protocolo inicial únicamente podrá clasificarse la situación del paciente en uno de los grupos mencionados

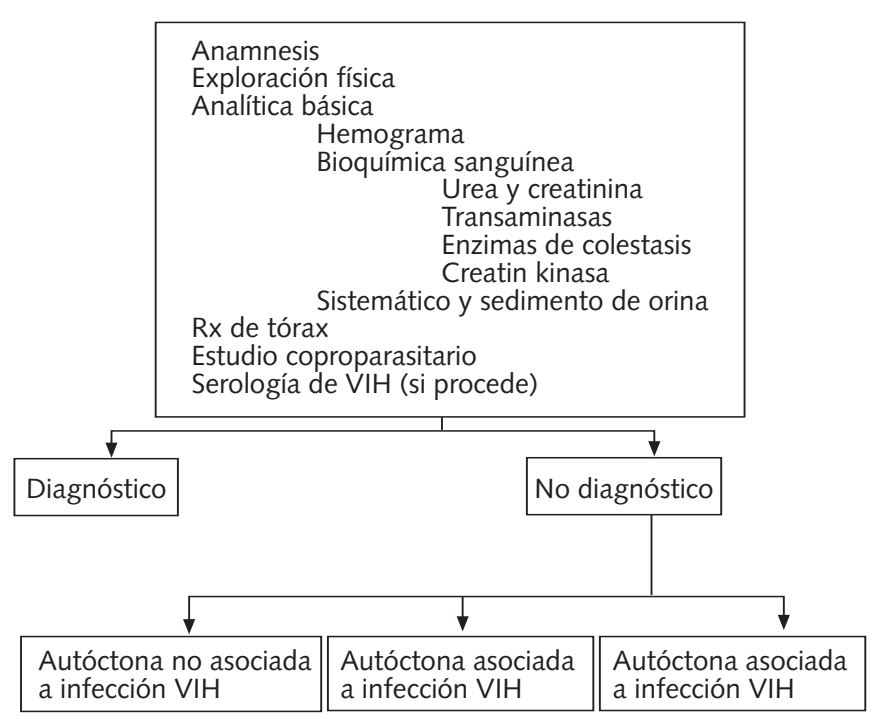

Fig. 1. Manejo diagnóstico inicial ante un paciente con eosinofilia.

En el paciente que no ha viajado a regiones tropicales y no está infectado por el VIH, deberá considerarse la realización de varias pruebas complementarias para descartar algunas entidades específicas, ya mencionadas previamente. Una ayuda diagnóstica consiste en considerar si el paciente presenta datos localizadores únicos (p. ej. pulmonares, cutáneas, etc), múltiples datos localizadores o no existen otras manifestaciones clínicas (Fig. 2).

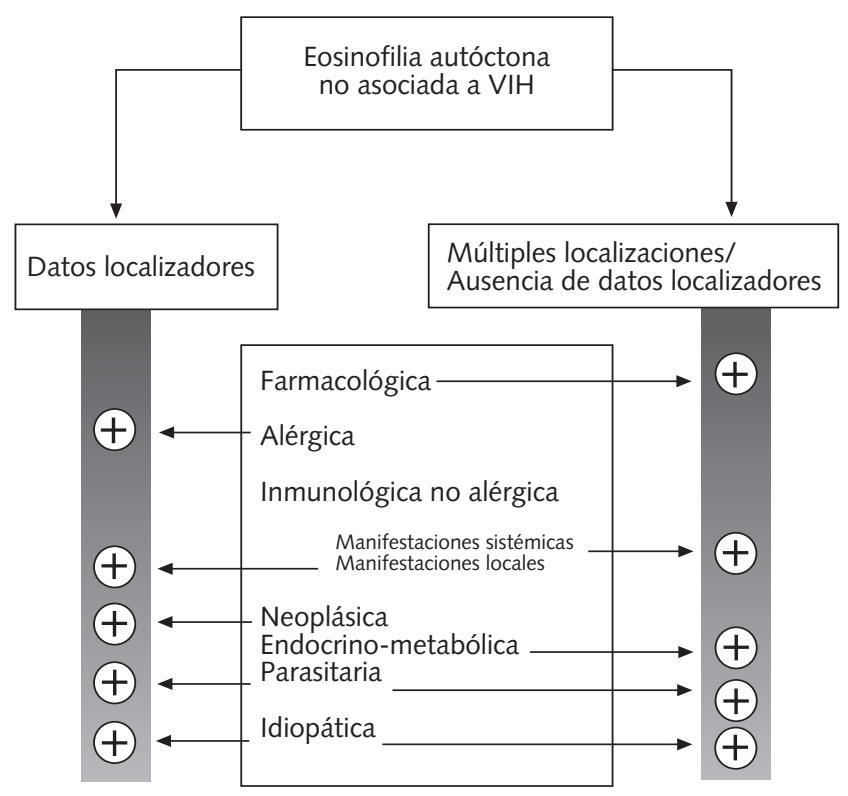

Fig. 2. Orientación diagnóstica en el paciente con eosinofilia autóctona no asociada a infección VIH. 
En el paciente que no ha viajado a regiones tropicales y está infectado por el VIH deberá reevaluarse de forma específica el uso de fármacos (cotrimoxazol, tuberculostáticos, antirretrovirales), la presencia de lesiones cutáneas (foliculitis eosinofílica, DRESS) y el estudio parasitario tanto coprológico (Isospora belli, Strongyloides stercoralis) como serológico (Strongyloides sp). En ausencia de datos sugerentes, deberá controlarse periódicamente el hemograma sin necesidad de estudios más agresivos.

Finalmente, en el paciente en el que se sospeche patología importada se plantean dos situaciones diferentes (i) el paciente en el que existen datos localizadores, en cuyo caso deberán realizarse pruebas dirigidas tanto de imagen (ecografía, TAC, RMN), obtención de líquidos biológicos (estudio de esputo, punción lumbar, toracocentesis), o toma de muestras de tejidos (p. ej. piel, hígado, recto o médula ósea) y (ii) si no existen datos de localización, en cuyo caso deberán realizarse estudios de despistaje que incluyen técnicas específicas de estudio de heces (p. ej. técnica de Baermann), obtención de contenido duodenal (mediante endoscopia o Entero-Test ${ }^{\circledR}$ ), estudios serológicos dirigidos (p. ej. empleando antígenos de Schistosoma sp., Strongyloides sp. o Dirofilaria sp.), evaluación de hemoparásitos a diferentes horas del día y con técnicas específicas (test de Knott), estudio de orina de 24 horas y, eventualmente, pruebas de provocación (test de Mazzotti).

\section{CONSECUENCIAS PATOLÓGICAS DE LA EOSINOFILIA}

De forma indirecta, la eosinofilia puede indicar la presencia de una parasitosis que comprometa la vida del paciente y de esta forma ocasionar consecuencias nocivas. Así, aunque el diagnóstico específico de todas las parasitosis es importante para efectuar un tratamiento etiológico, sin lugar a dudas, el principal parásito que debe descartarse es Strongyloides stercoralis. Esta afirmación se debe a las características de su ciclo biológico, su persistencia durante décadas en el organismo y la posibilidad, relativamente elevada (59), de desarrollar un sindrome de hiperinfección cuando concurre una situación de inmunodepresión (p. ej. uso de corticoides a dosis elevadas o coinfección por VIH).

Por otro lado, los eosinófilos son capaces de sintetizar y liberar directamente un gran número de mediadores proinflamatorios. Aunque ha sido controvertido, en la actualidad existen datos suficientes para afirmar que los eosinófilos (a través de sus mediadores) juegan un papel importante en el control de la fase larvaria de las parasitosis $(60,61)$. Por ello, la eosinofilia inducida por las helmintosis puede interpretarse como un mecanismo de defensa del hospedador. Sin embargo, estos mediadores inflamatorios liberados por los eosinófilos, pueden lesionar diversas estructuras del propio hospedador, siendo muy característica la afectación cardiaca (62). La lesión de las estructuras del corazón en la eosinofilia puede adoptar dos formas polares: fibrosis endomiocárdica de Davies (en áreas tropicales) o Loeffler (en regiones templadas) y miocarditis eosinofílica (62). Independientemente de la forma clínica que adopte, la afectación cardiaca por la eosinofilia inducida por parásitos ha sido documentada en estudios epidemiológicos así como en trabajos experimentales clínicos o en modelos animales (63-66). Sin embargo, en la actualidad quedan muchos aspectos no aclarados acerca de esta asociación, como la relación entre la afectación cardiaca eosinófila con el tipo de parásito causal, la importancia del grado de eosinofilia o la presencia de casos subclínicos.

\section{MANEJO TERAPÉUTICO DE UN PACIENTE CON EOSINOFILIA}

Evidentemente, la mejor forma de corregir una eosinofilia es el tratamiento etiológico. En este sentido, la retirada de la medicación responsable (eventualmente asociada a corticosteroides en formas graves) hará desaparecer la eosinofilia debidas a fármacos; la corrección del defecto vitamínico normalizará el hemograma en la anemia megaloblástica o la administración hormonal corregirá las alteraciones de la insuficiencia suprarrenal.

El manejo de la eosinofilia producida por helmintos requiere algunas consideraciones especiales. Así, en la práctica todas las enfermedades tratables por helmintos responden a uno o varios de estos fármacos: albendazol, ivermectina, praziquantel, mebendazol y triclabendazol (67-69). La dietilcarbamacina, que todavía se indica en muchos textos actuales como tratamiento de las filariosis, además de sus importantes efectos secundarios, presenta un problema práctico: su dificultad de obtención por métodos convencionales. El praziquantel es el fármaco de elección en las cestodosis por adultos (teniosis, difilobotriosis e infección por Hymenolepis sp) así como en las trematodosis (esquistosomosis, paragonimosis, etc). En las cestodosis por formas larvarias (hidatidosis, cisticercosis) el tratamiento farmacológico tiene un papel colateral. En los casos en los que está indicado, el albendazol es el que presenta una actividad mayor en las equinococosis $(70,71)$, mientras que el praziquantel es más eficaz en la cisticercosis $(72,73)$. Las geohelmintosis (infecciones por Trichuris, Ascaris y uncinarias) y otras nematodosis intestinales (Enterobius vermicularis) responden adecuadamente a mebendazol o albendazol, siendo en general más eficaz el albendazol que el mebendazol (con excepción de la infección por Trichuris en la que sucede lo contrario) $(70,74)$. El albendazol también es útil en el tratamiento de la toxocariosis (75), la triquinelosis (76) y la infección por Ancylostoma caninum o braziliensis (larva cutánea migrans) (77). La ivermectina constituye el fármaco de elección de la mayor parte de las filariosis (linfáticas, oncocercosis, loaosis e infecciones por Mansonella streptocerca y Mansonella ozzardi) con excepción de Mansonella perstans (que se trata con mebendazol de forma prolongada) (78-80). La ivermectina también constituye el fármaco de elección en la infección por Strongyloides stercoralis (81) y es útil en el tratamiento de la larva cutánea migrans (77). Finalmente el triclabendazol es el fármaco actual de elección en el tratamiento de la fasciolosis (82). La baja toxicidad de estos fármacos, su comodidad de administración y el efecto sinérgico de algunas combinaciones (p. ej. ivermectina y albendazol en las filariosis linfáticas) (78) han sugerido su empleo empírico en situaciones en las que, aunque no se haya demostrado una helmintosis, se plantee esta posibilidad (83) o antes de efectuar el diagnóstico de síndrome hipereosinofílico (38).

En otras ocasiones, únicamente puede realizarse un tratamiento patogénico, disminuyendo el número de eosinófilos, y de esta forma su potencial patógeno. Los corticosteroides constituyen el fármaco habitual en el manejo de las eosinofilias relacionadas con fenómenos inmunes o idiopáticas. El mecanismo de acción de estos fármacos es doble, ya que disminuyen la eosinofilopoyesis y promueven la eliminación de eosinófilos al incrementar la apoptosis y secuestro de estas células (84). 


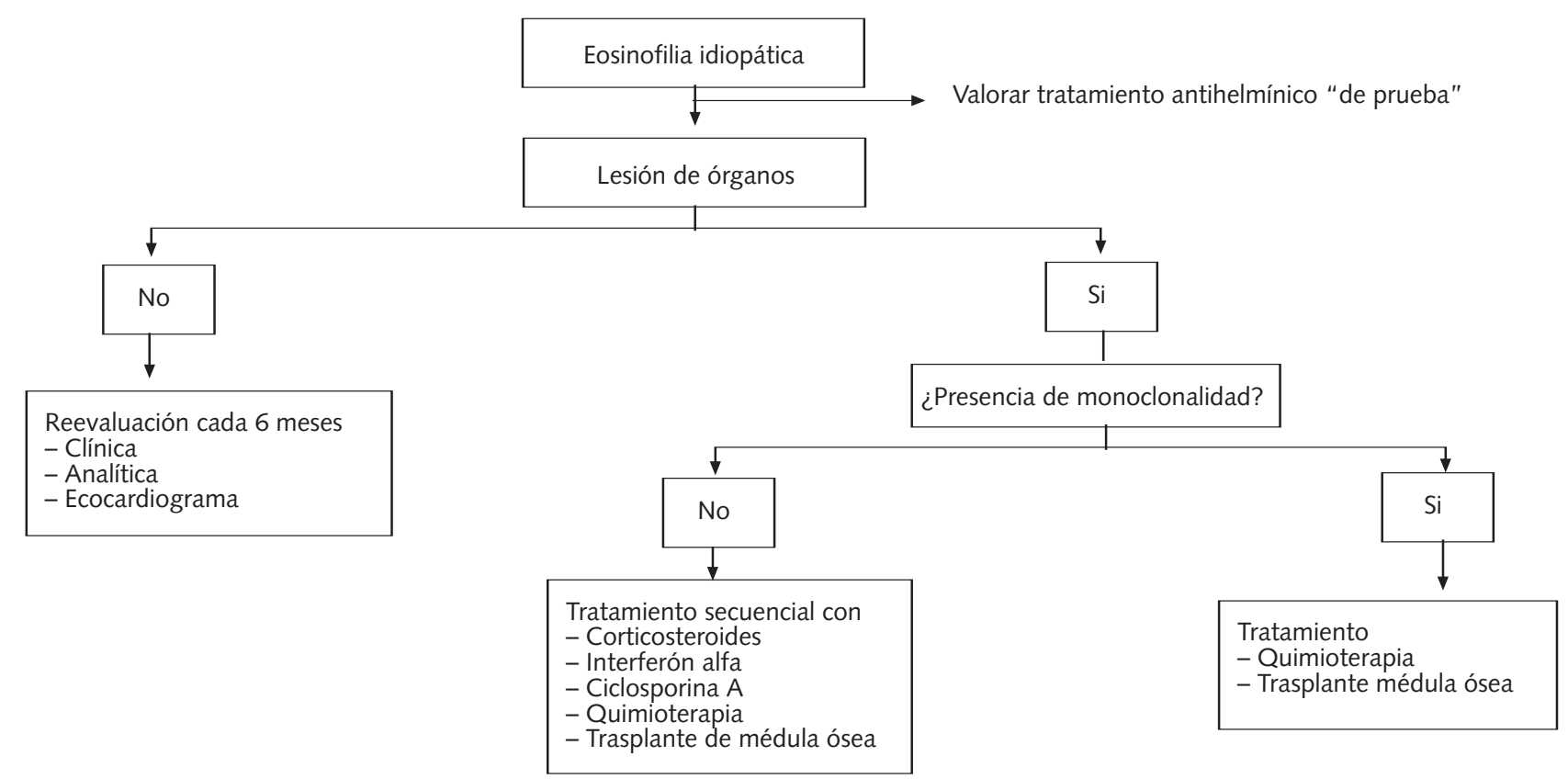

Fig. 3. Orientación diagnóstica en el paciente con eosinofilia autóctona no asociada a infección VIH.

El manejo inicial de la eosinofilia idiopática (Fig. 3) requiere, además del eventual tratamiento antihelmítico (ivermectina $400 \mu \mathrm{g} / \mathrm{Kg}$ y albendazol $400 \mathrm{mg}$ en dosis única), la valoración de afectación de órganos. Si no existe afectación tisular, es aconsejable el seguimiento cada 6 meses, replanteando el diagnóstico y realizando un ecocardiograma de control (ya que la lesión cardiaca puede progresar insidiosamente, sin relación con la eosinofilia sanguínea). Si existe afectación orgánica, es importante estudiar la presencia de datos de clonalidad (anomalías cromosómicas, poblaciones con idéntico fenotipo, hibridación in situ, etc.). En presencia de monoclonalidad, el tratamiento será el de la neoplasia hematológica responsable (quimioterapia y/o transplante de médula ósea). Específicamente en los casos de síndrome hipereosinofílico idiopático en los que se compruebe la translocación t $(1,4)$ (q44,q12) está indicado el tratamiento con imatinib $(39,85)$. Si

\section{Bibliografía}

1. Rothenberg ME. Eosinophilia. N Engl J Med 1998; 338: 1592-600.

2. Brigden ML. A practical workup for eosinophilia. You can investigate the most likely causes right in your office. Postgrad Med 1999; 105; 193-210.

3. Keystone JS, Philpott J. Eosinophilia in travelers and immigrants. En Strickland GT (ed) : Hunter's Tropical Medicine ( $7^{\mathrm{a}}$ ed) Philadelphia, WB Saunders 1991; 1038-42.

4. Leder K, Weller PF. Eosinophilia and helminthic infections. Baillierès Best Pract Res Clin Haematol 2000; 13: 301-17.

5. Maidment I, William C. Drug-induced eosinophilia. Pharmac J 2000; 264: 71-6.

6. Myers RP, McLaughlin K, Hollomby DJ. Acute interstitial nephritis due to omeprazole. Am J Gastroenterol 2001; 96: 3428-31.

7. Kanny G, Renaudin JM, Lecompte T, Moneret-Vautrin DA. Chloroquine hypersensitivity syndrome. Eur J Intern Med 2002; 13: 75-6. no se detecta clonalidad, el tratamiento inicial debe realizarse con prednisona $(1 \mathrm{mg} / \mathrm{kg} / \mathrm{día})$ en dosis descendentes hasta alcanzar la mínima dosis necesaria. Son factores que predicen una buena respuesta a los corticoides la asociación con angioedema, la elevación de IgE y la eosinopenia prolongada tras una única dosis de corticoides (86). En aquellos casos en los que no existe respuesta a los corticoides puede emplearse $\alpha$ interferón $(87,88)$ o ciclosporina (89). Finalmente en casos muy agresivos debe intentarse el uso de quimioterapia (84) y eventualmente transplante de médula ósea (90-92).

\section{AGRADECIMIENTOS}

Este trabajo ha sido desarrollado, en parte, gracias al proyecto FIS 01/685.

8. Mathias S, Schaaf LW, Sonntag A. Eosinophilia associated with olanzapine. J Clin Psychiatry 2002; 63: 246-7.

9. Voutsadakis IA. Fludarabine-induced eosinophilia: case report. Ann Hematol 2002; 81: 292-3.

10. Mofredj A, Boudjema H, Cadranel JF. Norfloxacin-induced eosinophilia in a cirrhotic patient. Ann Pharmacother 2002; 36: 1107-8.

11. Vlahakis NE, Aksamit TR. Diagnosis and treatment of allergic bronchopulmonary aspergillosis. Mayo Clin Proc 2001; 76: 930-8.

12. Conron M, Beynon HLC. Churg-Strauss syndrome. Thorax 2000; 55: $870-7$.

13. Masi AT, Hunder GG, Lie JT, et al. The American College of Rheumatology 1990 criteria for the classification of Churg-Strauss syndrome (allergic granulomatosis and angiitis). Arthritis Rheum 1990; 33: 1094-100. 
14. Doyle JA, Ginsburg WW. Eosinophilic fasciitis. Med Clin North Am 1989; 73: 1157-66

15. Jederlinic PJ, Sicilian L, Gaensler EA. Chronic eosinophilic pneumonia. A report of 19 cases and a review of the literature. Medicine (Baltimore) 1988; 67: 154-62.

16. Losa Garcia JE, Mateos Rodriguez F, de la Calle B, Rodriguez Encinas A, Sanchez Sanchez R, Perez Arellano JL. Neumonía eosinófila aguda en una gestante. Arch Bronconeumol 1997; 33: 306-8.

17. Zhang JZ, Zhang CG, Chen JM. Thirty-five cases of Kimura's disease (eosinophilic lymphogranuloma). Br J Dermatol 1998; 13: 542-3.

18. Seregard S. Angiolymphoid hyperplasia with eosinophilia should not be confused with Kimura's disease. Acta Ophthalmol Scand 2001; 79: 91-3.

19. Weiss G, Shemer A, Confino Y, Kaplan B, Trau H. Wells' syndrome: report of a case and review of the literature. Int J Dermatol 2001; 40: 148-52.

20. Chikama R, Hosokawa M, Miyazawa T, Miura R, Suzuki T, Tagami H Nonepisodic angioedema associated with eosinophilia: report of 4 cases and review of 33 young female patients reported in Japan. Dermatology 1998; 197: 321-5.

21. Butterfield JH, Leiferman KM, Gleich GJ. Nodules, eosinophilia, rheumatism, dermatitis and swelling (NERDS): a novel eosinophilic disorder. Clin Exp Allergy 1993; 23: 571-80

22. Mazanec DJ. Eosinophilic fasciitis and pernicious anemia with thyroid antibodies. J Rheumatol 1982; 9: 742-3.

23. Remacha Tomey B, Palau A, Colom J, Ripolles V. Gastroenteritis eosinofilica. An Med Interna (Madrid) 1998; 15: 554-9.

24. Walsh RE, Gaginella TS. The eosinophil in inflammatory bowel disease. Scand J Gastroenterol 1991; 26: 1217-24.

25. Weller PF. Eosinophilic meningitis. Am J Med 1993; 95: 250-3.

26. Itano NM, Malek RS. Eosinophilic cystitis in adults. J Urol 2001; 165: 805-7.

27. Vanherweghem JL, Leon M, Goldman M, Lietar N, Gamar N, Thayse C. Membrane-related eosinophilia in hemodialysis. Kidney Int Suppl 1988; 24: S73-4.

28. Ejaz AA, Fitzpatrick PM, Durkin AJ, et al. Pathophysiology of peritoneal fluid eosinophilia in peritoneal dialysis patients. Nephron 1999; 81: 125-30.

29. Grimbacher B, Holland SM, Gallin JI et al. Hyper-IgE syndrome with recurrent infections- an autosomal dominant multisystem disorder. $\mathrm{N}$ Engl J Med 1999; 340: 692-702.

30. Aleman K, Noordzij JG, de Groot R, van Dongen JJ, Hartwig NG. Reviewing Omenn syndrome. Eur J Pediatr 2001; 160: 718-25.

31. Samoszuk M. Eosinophils and human cancer. Histol Histopathol 1997; 12: 807-12.

32. Beishuizen A, Vermes I, Hylkema BS, Haanen C. Relative eosinophilia and functional adrenal insufficiency in critically ill patients. Lancet 1999; 353: 1675-6.

33. Thadhani RI, Camargo CA Jr, Xavier RJ, Fang LS, Bazari H. Atheroembolic renal failure after invasive procedures. Natural history based on 52 histologically proven cases. Medicine (Baltimore) 1995; 74: 350-8.

34. López Vélez R. Protocolo diagnóstico de las eosinofilias causadas por parásitos. Medicine (Madrid) 1998; 7: 3783-6.

35. Elcuaz R, Bolaños M, Guerra L, de Fuentes I, Lafarga B. Eosinofilia persistente y hallazgo parasitológico en un adulto. Enferm Infecc Microbiol Clin 1998; 16: 245-6.

36. Fauci AS, Harley JB, Roberts WC et al. The idiopathic hypereosinophilic syndrome. Clinical, pathophysiologic and therapeutic considerations. Ann Intern Med 1982; 97: 78-92.

37. Lin AY, Nutman TB, Kaslow D et al. Familial eosinophilia: clinical and laboratory results on a U.S. kindred. Am J Med Genet 1998; 76: 229-37.

38. Brito-Babapulle F. The eosinophilias including the idiopathic hypereosinophilic síndrome. Br J Haematol 2003; 121: 203-23.

39. Cools J, DeAngelo DJ, Gotlib J, et al. A tyrosine kinase created by fusion of the PDGFRA and FIP1L1 genes as a therapeutic target of imatinib in idiopathic hypereosinophilic syndrome. N Engl J Med 2003; 348, 1201-14

40. Cohen AJ, Steigbigel RT. Eosinophilia in patients infected with human immunodeficiency virus. J Infect Dis 1996; 174: 615-8.

41. Tietz A, Sponagel L, Erb P, Bucher H, Battegay M, Zimmerli W. Eosinophilia in patients infected with the human immunodeficiency virus. Eur J Clin Microbiol Infect Dis 1997; 16: 675-7.

42. Skiest DJ, Keiser P. Clinical significance of eosinophilia in HIV-infected individuals. Am J Med 1997; 102: 449-53

43. Claudio GA, Martin AF, de Dios Perrino S, Velasco AA. DRESS syndrome associated with nevirapine therapy.. Arch Intern Med 2001; 161 : 2501-2.
44. Verdon R, Six M, Rousselot P, Bazin C. Efavirenz-induced acute eosinophilic hepatitis. J Hepatol 2001; 34: 783-5.

45. Patey O, Lacheheb A, Dellion S, Zanditenas D, Jungfer-Bouvier F, Lafaix C. A rare case of cotrimoxazole-induced eosinophilic aseptic meningitis in an HIV-infected patient. Scand J Infect Dis 1998; 30: 530-1.

46. Casado JL, Piedrola G. Adrenal insufficiency in patients with AIDS: when to suspect it and how to diagnose it. AIDS Patient Care STDS 1997; 11: 339-43.

47. Simpson-Dent S, Fearfield LA, Staughton RC. HIV associated eosinophilic folliculitis--differential diagnosis and management. Sex Transm Infect 1999; 75: 291-3.

48. Harley WB, Blaser MJ. Disseminated coccidioidomycosis associated with extreme eosinophilia. Clin Infect Dis 1994; 18: 627-9.

49. DeHovitz JA, Pape JW, Boncy M et al. Clinical manifestations and therapy of Isospora belli infection in patients with the acquired immunodeficiency syndrome. N Engl J Med 1986; 315: 87-90.

50. Cuffari C, Oligny L, Seidman EC. Dientamoeba fragilis masquerading as allergic colitis. J Pediatr Gatroenterol Nutr 1998; 26: 16-20.

51. van der Enden E, Praet M, Joos R et al. Eosinophilic myositis resulting from sarcocystosis. J Trop Med Hyg 1995; 98: 273-6.

52. Zaki M, Daoud AS, Pugh RN, al-Ali F, al-Mutairi G, al-Saleh Q. Clinical report of Blastocystis hominis infection in children. J Trop Med Hyg 1991; 94: 118-22.

53. Wilson ME, Weller PF. Eosinophilia. En Guerrant RL, Walker DH, Weller PF (eds): Tropical infectious diseases. Principles, pathogens and practice ( $1^{\text {a }}$ ed) Philadelphia, Churchill Livingstone 1999; 1400-19.

54. Sato YJ, Kobayashi J, Toma H, Shiroma Y. Efficacy of stool examination for detection of Strongyloides infection. Am J Trop Med Hyg 1995; 53: 248-50.

55. Sanford CE, Jong EC. The eosinophilic patient with suspected parasitic infection. En: Jong EC, McMullen R. The travel and tropical medicine manual. $5^{\mathrm{a}}$ ed. WB Saunders 2003: 592-603.

56. Wolfe MS. Eosinophilia in the returning traveler. Med Clin North Am 1999; 83: 1019-32.

57. Libman MD, McLean JD, Gyorkos TW. Screening for schistosomosis, filariasis and strongyloidiasis among expatriates returning from the tropics. Clin Infect Dis 1993; 17: 353-9.

58. Nutman TB, Ottesen EA, Ieng S, et al. Eosinophilia in Southeast Asian refugees: Evaluation at a referal center. J Infect Dis 1987; 155: 309-12.

59. Milder J, Walzer P, Kilgore D, Rutheford I, Klein M. Clinical features of Strongyloides stercoralis in a endemic area of the United States. Gastroenterology 1981; 80: 1481-8.

60. Behm CA, Ovington KS. The role of eosinophils in parasitic helminth infections: insights from genetically modified mice. Parasitol Today 2000; 16: 202-9.

61. Meusen ENT, Balic A. Do eosinophils have a role in the killing of helminth parasites?. Parasitol Today 2000; 16: 95-101.

62. Touze JE, Fourcade L, Heno P, Mafart B, Mourot S. The heart and the eosinophil. Med Trop (Mars) 1998; 58 (Suppl 4): 459-64.

63. Rutakingirwa M, Ziegler JL, Newton R, Freers J. Poverty and eosinophilia are risk factors for endomyocardial fibrosis (EMF) in Uganda. Trop Med Int Health 1999; 4: 229-35.

64. Andy JJ, Ogunowo PO, Akpan NA, Odigwe CO, Ekanem IA, Esin RA Helminth associated hypereosinophilia and tropical endomyocardial fibrosis (EMF) in Nigeria. Acta Trop 1998; 69: 127-40.

65. Paolocci N, Sironi M, Bettini M, et al. Immunopathological mechanisms underlying the time-course of Trichinella spiralis cardiomyopathy in rats. Virchows Arch 1998; 432: 261-6.

66. Arima M, Kanoh T. Eosinophilic myocarditis associated with dense deposits of eosinophil cationic protein (ECP) in endomyocardium with high serum ECP. Heart 1999; 81: 669-71.

67. Liu LX, Weller PW. Antiparasitic drugs. N Engl J Med 1996; 334 1178-84.

68. de Silva N, Guyatt H, Bundy D. Anthelmintics. A comparative review of their clinical pharmacology. Drugs 1997; 53: 769-88.

69. Stephenson I, Wiselka M. Drug treatment of tropical parasitic infections: recent achievements and developments. Drugs 2000; 60: 985-95.

70. Venkatesan. Albendazole. J Antimicrob Chemother 1998; 41, 145-7.

71. Luchi S, Vincenti A, Messina F, Parenti M, Scasso A, Campatelli A. Albendazole treatment of human hydatid tissue. Scand J Infect Dis 1997; 29: 165-7.

72. Carpio A. Neurocysticercosis: un update. Lancet Infect Dis 2002; 2 751-62.

73. García HH, Evans CAW, Nash TE et al. Current consensus guidelines for treatment of neurocysticercosis. Clin Microbiol Rev 2002; 15: 747-56. 
74. Cowden J, Hotez P. Mebendazole and albendazole treatment of geohelminth infections in children and pregnant women. Pediatr Infect Dis J 2000; 19: 659-60.

75. Pawlowski Z. Toxocariasis in humans: clinical expression and treatment dilemma. J Helminthol 2001; 75: 299-305.

76. Capo V, Despommier DD. Clinical aspects of infection with Trichinella spp. Clin Microbiol Rev 1996; 9: 47-54.

77. Caumes E. Treatment of Cutaneous Larva Migrans. Clin Infect Dis 2000; 30: 811-14.

78. Melrose WD. Lymphatic filariasis: new insights into an old disease. Int J Parasitol 2002; 32: 947-60.

79. Hoerauf A, Buttner DW, Adjei O, Pearlman E. Onchocerciasis. BMJ 2003; 326: 207-10.

80. Ottesen EA, Campbell WC. Ivermectin in human medicine. J Antimicrob Chemother 1994; 34: 195-203.

81. Zaha O, Hirata T, Kinjo F, Saito A. Strongyloidiasis. Progress in diagnosis and treatment. Intern Med 2000; 39: 695-700.

82. Arjona R, Riancho JA, Aguado JM, Salesa R, Gonzalez-Macias J. Fascioliasis in developed countries: a review of classic and aberrant forms of the disease. Medicine (Baltimore) 1995; 74: 13-23.

83. Moliner S., Chaudier B, Kraemer P et al. Diagnostic and treatment of hypereosinophilia upon return from the tropics; 102 patients. Medecine Tropicale: Revue Du Corps de Sante Colonial 1998; 58: 499-502.
84. Weller, PF, Bubley, GJ. The idiopathic hypereosinophilic syndrome Blood 1994; 83: 2759-79.

85. Schwartz RS. The hypereosinophilic síndrome and the biology of cancer. N Engl J Med 2003; 348: 1199-200.

86. Parrillo, JE, Fauci, AS, Wolff, SM. Therapy of the hypereosinophilic syndrome. Ann Intern Med 1978; 89: 167-72.

87. Bockenstedt, PL, Santinga, JT, Bolling, SF. Alpha-interferon treatment for idiopathic hypereosinophilic syndrome. Am J Hematol 1994; 45: 248-51.

88. Canonica GW, Passalacqua G, Pronzato C, et al. Effective long-term alpha-interferon treatment for hypereosinophilic syndrome. J Allergy Clin Immunol 1995; 96: 131-3.

89. Nadarajah S, Krafchik B, Roifman C, Horgan-Bell C. Treatment of hypereosinophilic syndrome in a child using cyclosporine: Implication for a primary T-cell abnormality. Pediatrics 1997; 99: 630-6.

90. Esteva-Lorenzo FJ, Meehan KR, Spitzer TR, Mazumder A. Allogeneic bone marrow transplantation in a patient with hypereosinophilic syndrome. Am J Hematol 1996; 51: 164-5.

91. Sigmund DA, Flessa HC. Hypereosinophilic syndrome: Successful allogeneic bone marrow transplantation. Bone Marrow Transplant 1995; 15: 647-8.

92. Fukushima T, Kuriyama K, Ito H, et al. Successful bone marrow transplantation for idiopathic hypereosinophilic syndrome. Br J Haematol 1995; 90: 213-5. 